\title{
The Clinical Effect of Seasonal Flu Vaccination on Health-Related Quality of Life
}

\author{
Yusuke Yoshino',2 \\ Yoshitaka Wakabayashi' \\ Takatoshi Kitazawa' \\ 'Department of Internal Medicine, Teikyo \\ University School of Medicine, Tokyo, \\ Japan; ${ }^{2}$ Department of Microbiology, \\ Teikyo University School of Medicine, \\ Tokyo, Japan
}

Purpose: This study aimed to determine the clinical effect of seasonal flu vaccination in adult outpatients based on the effect on health-related quality of life (HRQOL).

Patients and Methods: We reviewed the clinical data of adult flu outpatients with mild symptoms who presented to the outpatient clinic of Teikyo University Hospital, Tokyo, Japan, from 2018 to 2020 winter season and were enrolled in the prospective observational study of the clinical efficacy of anti-flu agents (UMIN000034896). We evaluated influenza vaccination status, clinical symptoms, and the status of HRQOL as measured by Short Form$8^{\circledR}\left(\mathrm{SF}-8^{\circledR}\right)$ at first visit. The SF- $8^{\circledR}$ survey generated two-component summaries; a physical component summary and a mental component summary.

Results: The data of 79 patients were evaluated in this study. Of the 79 patients, 37 were vaccinated for influenza at least three weeks before contracting seasonal influenza. Not every patient needed to be hospitalized for treatment. There were no significant differences in clinical backgrounds between vaccinated patients and non-vaccinated patients. Univariate analysis showed influenza vaccination was significantly associated with higher scores on the physical component summary of HRQOL ( $\mathrm{p}=0.0011)$.

Conclusion: Influenza vaccinations would be clinically valuable for adult outpatients with seasonal flu and mild symptoms, because they can significantly inhibit the decrease of HRQOL due to influenza infection.

Keywords: influenza vaccination, health-related quality of life, 8-Item Short Form Health Survey, SF-8, patient-reported outcome

\section{Introduction}

Influenza patients exhibit upper and/or lower respiratory tract signs and symptoms, often accompanied by systemic illness symptoms, such as fever, headache, myalgia, and weakness. Despite being a self-limiting disease, influenza can also occur in the pandemic form. Influenza pandemics often lead to serious health complications, deaths, as well as social and economic disruption. Due to health concerns for individuals and the potential for pandemics, influenza prevention, and control strategies, including vaccination, is available worldwide. Indeed, the United States Advisory Committee on Immunization Practices recommends annual influenza vaccination for all individuals six months of age and older. ${ }^{1}$ Inactivated influenza vaccines and a live attenuated vaccine are available in the US. Only the inactivated influenza vaccine is available in Japan. The available standard-dose inactivated influenza vaccine in Japan is a quadrivalent preparation of split virions that have been inactivated. This type of vaccine is one of the major vaccines globally, approved due to its safety and clinical effectiveness. A population-based
Correspondence: Yusuke Yoshino Department of Internal Medicine, Teikyo University School of Medicine, 2-11-I

Kaga, Itabashi-ku, Tokyo, 173-8606, Japan $\mathrm{Tel}+8 \mathrm{I}-3-3964-121 I$

Email yyoshino@med.teikyo-u.ac.jp 
study in the United States assessed the effect of influenza vaccination on disease severity among hospitalized patients during the 2013 to 2014 influenza season. In a season in which vaccine viruses were antigenically similar to circulating viruses, influenza vaccination was associated with a reduced odds of in-hospital death among patients aged 18 to 64 years of age. ${ }^{2}$ However, almost all seasonal flu patients are outpatients with mild symptoms such as fever or feeling feverish/chills, cough, sore throat, runny or stuffy nose, muscle aches, joint aches, headaches, fatigue, vomiting, and diarrhea. Furthermore, there are few clinical reports on the clinical benefits of flu vaccination in outpatients with mild symptoms. ${ }^{3,4}$ In addition, there have been no reports on the benefit of flu vaccination evaluated by patient-reported outcomes for outpatients with mild symptoms. The aim of this research study was to determine the clinical effect of seasonal flu vaccination in adult outpatients based on the effect on Health-Related Quality of Life (HRQOL), as reported by patients.

\section{Methods}

This study is a sub-analysis of the prospective observational study, approved by the ethics committee of Teikyo University (No. TUIC-COI 18-0760), which examined the therapeutic effect of anti-flu agents for seasonal flu. This trial enrolled all influenza patients visiting the outpatient clinic of the department of internal medicine of Teikyo University Hospital, Tokyo, Japan, between November 2018 and February 2020. Written informed consent was obtained from all study participants. Diagnosis with influenza was based on a positive influenza rapid antigen test result (Fujirebio Espline Influenza A\&B-N, Tokyo, Japan, sensitivity and specificity: 91\% and $97 \%$, respectively) in addition to the presentation of fever and at least one of the other signs and symptoms of influenza. ${ }^{5}$ These include muscle pain, chills, sweating, headache, dry cough, fatigue, nasal congestion, disorientation, hypotension, and respiratory failure, without other focal infection signs. Fever was defined as an axillary temperature of $\geq 37.0^{\circ} \mathrm{C}$, and any patient febrile for $\geq 48$ hours on the first hospital visit was excluded from this trial. Demographic and clinical data, including age, sex, and maximum body temperature after disease onset, and time course of fever, were collected on the first visit.

Moreover, we noted symptoms such as cough, sore throat, nasal discharge, headache, muscle pain, joint pain, nausea/vomiting, diarrhea, body temperature, and influenza type (influenza A or B). We also assessed the general
HRQOL based on the Japanese version of the 8-Item Short Form Health Survey (SF-8), a shortened version of the form used in the original Medical Outcome Study. ${ }^{6}$ The SF-8 is an HRQOL assessment that consists of eight scales (PF - physical functioning, RP - role physical, BP bodily pain, GH - general health, VT - vitality, SF - social functioning, RE - role emotional, and $\mathrm{MH}$ - mental health) and generates two summary measures, namely a physical component summary (PCS) and a mental component summary (MCS). To evaluate the association between vaccination status and these clinical features, including the score of HRQOL, the status of fever, and the presence of other clinical symptoms, we used the Student's $t$-test and chi-square test as univariate analyses to analyze continuous and categorical data, as appropriate. All p-values were two-sided and considered statistically significant at $p<0.05$. StatFlex version 6 software (Artech Co., Osaka, Japan) was used for analysis.

\section{Results}

The data of 79 patients were evaluated. Of these, 37 patients were vaccinated at least 3 weeks before the onset of symptoms (vaccinated group), and 42 patients were not vaccinated (non-vaccinated group). The clinical features of these two groups are described in Table 1 . There were no significant differences in the backgrounds of the groups, including age, sex, phenotype, comorbidities, and smoking status. When reviewing the summaries generated by the SF-8, PCS of HRQOL was significantly lower in the non-vaccinated group than the vaccinated group (vaccinated group: $37.73 \pm 9.74$ vs non-vaccinated group: $29.55 \pm 11.42, \mathrm{p}=0.0011)$. In contrast, there was no significant difference in MCS of HRQOL between the two groups (vaccinated group: $51.23 \pm 8.55$ vs non-vaccinated group: 50.13 $\pm 6.98, \mathrm{p}=0.2870$ ). (Table 1).

Regarding clinical symptoms, body temperature on admission and maximum body temperature of the vaccinated group were lower than those of the non-vaccinated ( $37.91 \pm 0.66$ vs $38.21 \pm 0.72$ degree Celsius, $\mathrm{p}=0.0551$ at first admission and $38.39 \pm 0.66$ vs $38.66 \pm 0.64$ degree Celsius, $\mathrm{p}=0.0709$ maximum temperature), though this was not statistically significant. There were also no statistically significant differences in the presence of symptoms other than fever (Table 1).

\section{Discussion}

Annual vaccination reduced mortality from influenza by $41 \%$ (95\% Confidence Interval (CI): $13-60 \%){ }^{7}$ Among 
Table I Clinical Backgrounds of Cases at First Visit, Clinical Symptoms, and Status of Health-Related Quality of Life

\begin{tabular}{|c|c|c|c|c|}
\hline & All $(n=79)$ & $\begin{array}{l}\text { Vaccinated Group } \\
(n=37)\end{array}$ & $\begin{array}{l}\text { Non-Vaccinated Group } \\
(n=42)\end{array}$ & $\mathbf{P}$ \\
\hline Age & $\begin{array}{l}42.41 \\
\pm 18.50\end{array}$ & $42.84 \pm 18.46$ & $42.02 \pm 18.75$ & 0.8467 \\
\hline Gender male & $46(58.23 \%)$ & $22(59.46 \%)$ & $24(57.14 \%)$ & 0.8350 \\
\hline Days after symptoms onset & $1.18 \pm 0.38$ & $1.16 \pm 0.37$ & $1.19 \pm 0.40$ & 0.7461 \\
\hline Phenot2ype A & $78(98.73 \%)$ & $36(97.30 \%)$ & $42(100 \%)$ & 1.0000 \\
\hline \multicolumn{5}{|l|}{ Backgrounds } \\
\hline Malignancy & $2(2.53 \%)$ & $0(0.00 \%)$ & $2(4.76 \%)$ & 0.4956 \\
\hline Congestive heart failure & $3(3.80 \%)$ & $2(5.41 \%)$ & $\mathrm{I}(2.38 \%)$ & 0.5971 \\
\hline Diabetes mellitus & $5(6.33 \%)$ & $3(8.11 \%)$ & $2(4.76 \%)$ & 0.6608 \\
\hline Chronic renal failure & $\mathrm{I}(\mathrm{I} .27 \%)$ & $0(0.00 \%)$ & $\mathrm{I}(2.38 \%)$ & 1.0000 \\
\hline Bronchial asthma & $5(6.33 \%)$ & $3(8.11 \%)$ & $2(4.76 \%)$ & 0.6608 \\
\hline Other airway diseases & $\mathrm{I}(\mathrm{I} .27 \%)$ & $0(0.00 \%)$ & $\mathrm{I}(2.38 \%)$ & 1.0000 \\
\hline Use of immunosuppressive agents & $2(2.53 \%)$ & $\mathrm{I}(2.70 \%)$ & $I(2.38 \%)$ & 1.0000 \\
\hline Immunosuppressive diseases & $2(2.53 \%)$ & $\mathrm{I}(2.70 \%)$ & $I(2.38 \%)$ & 1.0000 \\
\hline \multicolumn{5}{|l|}{ Symptoms } \\
\hline $\begin{array}{l}\text { Body temperature at first visit (degree } \\
\text { Celsius) }\end{array}$ & $38.07 \pm 0.70$ & $37.91 \pm 0.66$ & $38.21 \pm 0.72$ & 0.0551 \\
\hline $\begin{array}{l}\text { Maximum Body temperature (degree } \\
\text { Celsius) }\end{array}$ & $38.53 \pm 0.66$ & $38.39 \pm 0.66$ & $38.66 \pm 0.64$ & 0.0709 \\
\hline \multicolumn{5}{|l|}{ Presence of symptoms } \\
\hline Coughing & $72(91.14 \%)$ & $32(86.49 \%)$ & $40(95.24 \%)$ & 0.2430 \\
\hline Sore throat & $56(70.89 \%)$ & $24(64.86 \%)$ & $32(76.19 \%)$ & 0.2689 \\
\hline Nasal discharge & $63(79.75 \%)$ & $29(78.38 \%)$ & $34(80.95 \%)$ & 0.7764 \\
\hline Headache & $55(69.62 \%)$ & $22(59.46 \%)$ & $33(78.57 \%)$ & 0.0653 \\
\hline Muscle pain & $48(60.76 \%)$ & $21(56.76 \%)$ & $27(64.29 \%)$ & 0.4941 \\
\hline Joint pain & $54(68.35 \%)$ & $24(64.86 \%)$ & $30(71.43 \%)$ & 0.5314 \\
\hline Nausea/vomiting & $13(16.46 \%)$ & $7(18.92 \%)$ & $6(14.29 \%)$ & 0.5794 \\
\hline Diarrhea & $5(6.33 \%)$ & $3(8.11 \%)$ & $2(4.76 \%)$ & 0.6608 \\
\hline \multicolumn{5}{|l|}{ Health-related Quality of Life } \\
\hline Physical component summary & $\begin{array}{l}33.38 \\
\pm 11.36\end{array}$ & $37.73 \pm 9.74$ & $29.55 \pm 11.42$ & 0.0011 \\
\hline Mental component summary & $51.23 \pm 8.55$ & $50.13 \pm 6.98$ & $52.20 \pm 9.71$ & 0.2870 \\
\hline
\end{tabular}

those who had been vaccinated previously, mortality was reduced by $75 \%$ (95\% CI: 31-91\%), but among those being vaccinated for the first time, the reduction in mortality was only $9 \%(95 \%$ CI: $0-59 \%)$. The seasonal flu vaccine is designed to protect against the three or four influenza viruses that research indicates are most likely to spread and cause illness among people during the upcoming flu season. Each dose of seasonal influenza vaccine is formulated to contain the viruses representing the influenza $\mathrm{A}(\mathrm{H} 3 \mathrm{~N} 2)$ and $\mathrm{A}$ (H1N1) and influenza B strains, which are considered to most likely circulate in the upcoming influenza season. Even if the previous year's vaccine contained one or more antigens to be administered in the current year, annual immunization was necessary because immunity declined following vaccination. ${ }^{8}$ However, the Centers for Disease Control and Prevention showed flu vaccination coverage among adults $\geq 18$ years was only $45.3 \%$ during the 2018-19 flu season in the US. ${ }^{9}$ Among people $>2$ years old in Japan, the National Institute of Infectious Diseases showed a similar result; flu vaccination coverage was only about $45 \%$ during the $2017-18$ season. $^{10}$ The rate of flu vaccinations remained low. We believe it is important to increase this rate for society to benefit from flu vaccinations.

Flu vaccination has various positive additional effects. A case-control study in adults 40 years or older found that influenza vaccination was associated with a reduced rate of 
first acute myocardial infarction. ${ }^{11}$ Vaccinating younger adults appears to provide benefits for older adults in the same community. In a large nationwide sample of Medicare beneficiaries in the United States, vaccination of adults aged 18 to 64 years was inversely associated with influenza-related illness in individuals $\geq 65$ years of age. ${ }^{12}$ Though it was proven that the flu vaccine was highly effective in a social sense, it was difficult to demonstrate whether it was effective individually for younger adults. Almost all flu patients, especially younger adult patients, are outpatients with mild symptoms. Yet, most of the previous clinical research was focused on the efficacy in elderly people and in-patients with relatively severe symptoms. Therefore, we thought it was important to evaluate the clinical effect of flu vaccination for outpatients with mild symptoms. Indeed, there are a few published articles on the efficacy of influenza vaccine among young healthy adults, including this study. ${ }^{3,4}$ We evaluated the extent of HRQOL because seasonal flu is a highly contagious disease that leads to other indirect effects, including school absenteeism, loss of workplace productivity, and effects on HRQOL. ${ }^{13}$ This study was clinically valuable as it is the first report to reveal the clinical benefits of flu vaccination on HRQOL in adult outpatients with mild symptoms. We believe that our results will help young healthy adults learn about the importance of annual vaccination and encourage getting their annual flu shots.

This study was limited by its retrospective nature and relatively small sample size. There is a need for a large prospective cohort study to confirm the clinical benefits of flu vaccination for healthy adult outpatients using patientreported outcomes.

\section{Conclusion}

Influenza vaccinations would be clinically valuable for adult outpatients with seasonal flu and mild symptoms as they significantly inhibit the decrease of HRQOL due to influenza infection.

\section{Ethical Approval}

This study was approved by the Ethics Committee of the Teikyo University School of Medicine (No. TUIC-COI 180760), and written informed consent was obtained from all the study participants. Routine data for clinical purposes were used, and all patient information remained confidential in the study. All clinical research procedures were followed in accordance with the standards set forth in the Declaration of Helsinki principles of 1975, revised in 2013 (http://ethics.iit.edu/ecodes/node/3931).

\section{Acknowledgments}

We would like to thank Editage (www.editage.com) for English language editing.

\section{Disclosure}

The authors report no conflicts of interest in this work.

This research did not receive any specific grant from funding agencies in the public, commercial, or not-forprofit sectors.

\section{References}

1. Grohskopf LA, Alyanak E, Broder KR, et al. Prevention and control of seasonal influenza with vaccines: recommendations of the advisory committee on immunization practices - United States, 2019-20 influenza season. MMWR Recomm Rep. 2019;68:1-24. doi:10.15585/ mmwr.rr6803a1

2. Arriola C, Garg S, Anderson EJ, et al. Influenza vaccination modifies disease severity among community-dwelling adults hospitalized with influenza. Clin Infect Dis. 2017;65:1289-1297. doi:10.1093/cid/cix468

3. Grotto I, Mandel Y, Green MS, et al. Influenza vaccine efficacy in young, healthy adults. Clin Infect Dis. 1998;26:913-917. doi:10.1086/513934

4. Nichol KL, Lind A, Margolis KL, et al. The effectiveness of vaccination against influenza in healthy, working adults. $N$ Engl $J$ Med. 1995;333:889-893. doi:10.1056/NEJM199510053331401

5. De witte E, Goossens H, Ieven M. Evaluation of the ESPLINE ${ }^{\circledR}$ influenza A \& B-N assay for the detection of influenza A and B in nasopharyngeal aspirates. Eur $J$ Clin Microbiol Infect Dis. 2012;31:761-766. doi:10.1007/s10096-011-1372-1

6. Tokuda Y, Okubo T, Ohde S, et al. Assessing items on the SF-8 Japanese version for health-related quality of life: a psychometric analysis based on the nominal categories model of item response theory. Value Health. 2009;12:568-573. doi:10.1111/j.15244733.2008.00449.x

7. Ahmed AE, Nicholson KG, Nguyen-van-tam JS. Reduction in mortality associated with influenza vaccine during 1989-90 epidemic. Lancet. 1995;346:591-595. doi:10.1016/S0140-6736(95)91434-X

8. Bateman AC, Kieke BA, Irving SA, et al. Effectiveness of monovalent 2009 pandemic influenza A virus subtype H1N1 and 2010-2011 trivalent inactivated influenza vaccines in Wisconsin during the 2010-2011 influenza season. $J$ Infect Dis. 2011;207:1262-1269. doi:10.1093/infdis/jit020

9. Centers for Disease Control and Prevention. Flu vaccination coverage, United States, 2018-19 influenza season; 2019. Available from: https://www.cdc.gov/flu/fluvaxview/coverage-1819estimates.htm. Accessed March, 2020.

10. National Institute of Infectious Diseases. Infectious agents surveillance report (IASR); 2019 [updated December 2, 2019]. Available from: https://www.niid.go.jp/niid/ja/typhi-m/iasr-reference/2471-related-articles /related-articles-477/9231-477r04.html. Accessed March, 2020.

11. Siriwardena AN, Gwini SM, Coupland CA. Influenza vaccination, pneumococcal vaccination and risk of acute myocardial infarction: matched case-control study. CMAJ. 2010;182:1617-1623. doi:10.1503/cmaj.091891

12. Taksler GB, Rothberg MB, Cutler DM. Association of influenza vaccination coverage in younger adults with influenza-related illness in the elderly. Clin Infect Dis. 2015;61:1495-1503. doi:10.1093/cid/civ630

13. Thorrington D, Balasegaram S, Cleary P, et al. Social and economic impacts of school influenza outbreaks in England: survey of caregivers. J School Health. 2017;87:209-216. doi:10.1111/ josh. 12484 


\section{Publish your work in this journal}

The International Journal of General Medicine is an international, peer-reviewed open-access journal that focuses on general and internal medicine, pathogenesis, epidemiology, diagnosis, monitoring and treatment protocols. The journal is characterized by the rapid reporting of reviews, original research and clinical studies across all disease areas. The manuscript management system is completely online and includes a very quick and fair peer-review system, which is all easy to use. Visit http://www.dovepress.com/ testimonials.php to read real quotes from published authors.

Submit your manuscript here: https://www.dovepress.com/international-journal-of-general-medicine-journal 